Derecho y Realidad

Vol.14 - Núm. 27 • Enero - Junio de 2016

Págs. 125-136•ISSN:1692-3936

\title{
El sofisma de la participación juvenil. Una mirada crítica al discurso de la participación juvenil en Colombia*
}

\author{
The fallacy of youth participation. A critical \\ look at the speech of the youth participation in \\ Colombia
}

Néstor Moreno Huertas**

\section{Resumen}

La participación juvenil se ha presentado como uno de los aportes de avanzada dentro del discurso de oferta institucional, dirigido a los jóvenes. No obstante, este discurso desconoce el universo que le da significado a esta población, promoviendo una ruptura entre la participación y la movilización social. Lo anterior propicia un distanciamiento entre la población y las instituciones que pretenden promocionar la participación juvenil, ruptura que tiene sus raíces en la falta de interés de estas instituciones por incluir de manera real a la población juvenil en sus agendas políticas.

Fecha de aceptación: 12 de noviembre de 2015

Concepto de recepción: 13 de noviembre de 2015

Fecha de aprobación: 25 de noviembre de 2015

* Artículo corto, en el cual se presentan resultados y reflexiones originales, producto de la investigación que sustenta la tesis de Maestría en Derechos Humanos, titulada "Limitaciones conceptuales de la política pública de juventud en el ejercicio de los derechos humanos en el departamento de Boyacá".

** Abogado egresado de la Facultad de Derecho y Ciencias Sociales de la UPTC, magistrando en Derechos Humanos de la UPTC. Miembro oficial de la Organización No Gubernamental, Jóvenes Iberoamericanos. 


\title{
Palabras clave
}

Participación juvenil, derechos humanos, joven heredero, joven, juventud, movilización social.

\begin{abstract}
Youth participation has been presented as one of the new contributions within the speech of institutional offer towards young people. Nevertheless, this speech does not recognize the universe which gives significance to this population, promoting a rupture between participation and social mobilization. This situation enables a distancing between population and institutions which intend to promote youth participation. The rupture has its roots on the lack of interest of these institutions for including in a real way youth population in the political agenda.
\end{abstract}

\section{Keywords}

Youth participation, human rights, young heir, young, youth, social mobilization. 


\section{Introducción}

La reflexión que presentamos a continuación es parte de los resultados de la investigación "Limitaciones conceptuales de la política pública de juventud en el ejercicio de los derechos humanos en el departamento de Boyacá”. En dicho escrito se abordan los fenómenos relacionados con la juventud, en los aspectos históricos, sociológicos y políticos, que abarcan, desde los momentos históricos que construyen juventud, hasta los aspectos teóricos y sociológicos que explican a profundidad el fenómeno de esta población. De manera que teniendo en cuenta la característica principal de la reflexión que nos reúne, presentamos un artículo corto, en el cual se dará a conocer un análisis sobre cuestiones particulares del discurso institucional de la participación juvenil.

Este presentará tres momentos básicos, el primero tendrá un recorrido preciso por los momentos históricos que construyen juventud, con el fin de hacer memoria sobre la evolución de la población y sus complejidades. El segundo momento expondrá las características básicas de ese discurso institucional sobre participación juvenil, haciendo especial énfasis en el concepto del joven heredero y las consecuencias de esta invención sobre la participación juvenil; y por último, se cerrará el escrito con la conclusión, en donde se relacionan las categorías conceptuales que sustentan el espíritu del presente artículo.

En los últimos 30 años, América Latina se ha sumergido en discusiones en torno a los universos y contextos de la población juvenil. Sin embargo, para las instituciones y los jóvenes, este camino no ha sido fácil de recorrer, ya que ha sido un sendero lleno de dificultades, las cuales han logrado retrasar avances significativos en el reconocimiento y materialización de sus derechos.

Desde 1985, año en el cual se inicia la conmemoración del Día Internacional de la Juventud, la Cepal publica uno de los primeros estudios más representativos sobre la situación de la población juvenil en américa latina y el caribe (Cepal, 1985). Una de las principales conclusiones de dicho documento es que es necesario hacer un cambio de perspectiva en el tratamiento de la población juvenil. Invita a las instituciones a hacer una revisión profunda de las políticas públicas poblacionales, y cómo estas estaban atendiendo a la juventud en cada uno de sus territorios. Pero no todas las conclusiones eran precisamente alentadoras, sobre todo, tratándose de la precariedad en temas de derechos humanos. Uno de los muchos ejemplos se da a mediados de la década de los 80 , cuando se denunciaban no solo las consecuencias de la migración interna de la población rural hacia las ciudades en los países latinoamericanos, sino las dificultades que esto suponía para la materialización de los derechos humanos. 
Pero esto no era un problema que tuviera fundamento en causas propias de la naciente apertura económica, o de los fenómenos asociados a problemáticas de la época. Es decir, estos conflictos que amenazaban la vida de la población juvenil no eran causados únicamente por las particularidades de los años 80, pues estos tenían un desarrollo histórico, que solo hasta esta década fue posible llevar a las discusiones políticas internacionales.

\section{Metodología}

El presente artículo de reflexión se realizó como parte de las reflexiones que construyeron el trabajo de investigación titulado "Limitaciones conceptuales de la política pública de juventud en el ejercicio de los derechos humanos en el departamento de Boyacá", cuyo proceso de elaboración tuvo lugar en el contexto de trabajo de grado de la Maestría en Derechos Humanos. La metodología de investigación que se empleó en el presente artículo responde a la metodología de investigación documental, utilizada en la elaboración del trabajo antes mencionado, en la cual se consultaron tesis de maestría, libros, capítulos de libros, artículos científicos, documentos digitales y artículos periodísticos. Además, se hace un acercamiento crítico, con el fin de dar una resignificación al concepto de juventud desde perspectivas históricas y sociológicas, con el ánimo de construir una mirada crítica al discurso de participación juvenil en Colombia.

\section{Referencias sobre juventud}

Para fundamentar lo anterior, es preciso recordar que la cuestión de la población juvenil, a pesar de no ser nueva para la década de los 80 , en términos generales, sí lo es relativamente, si se observa desde una perspectiva histórica. Las investigaciones han reconocido que uno de los primeros antecedentes en la elaboración teórica de juventud se remite a las disertaciones de Juan Jacobo Rousseau. El teórico suizo, en su libro Emilio o de la educación, reflexionaba sobre la idea de juventud como un momento turbio, entre el estado de naturaleza de la niñez y el ciudadano en la etapa adulta del hombre. Convirtiéndose, así, en la principal fuente de la vertiente psicologista en los discursos sobre juventud.

Lo anterior es solo una muestra de los cientos de ejemplos que pueden rastrearse, tanto en el desarrollo teórico científico, como en el histórico, en donde se marcan puntos de inflexión que han transformado la vida de la población a lo largo de la historia. Como consecuencia de todos estos acontecimientos, la juventud ha tomado una relevancia que ha ido en aumento, sobre todo en las últimas tres décadas. Por otra parte, esta historia no ha sido del todo satisfactoria, ya que la inclusión de la 
población juvenil en las agendas políticas en la actualidad, se ha mantenido fuera del núcleo de las decisiones representativas, en cuanto a política social se refiere.

\section{Referentes históricos}

Retomando un poco la discusión histórica y teniendo en cuenta los consensos sobre el tema a los que han llegado los académicos tanto en Europa como en América Latina, recordaremos algunos momentos que han logrado construir juventud, alrededor de cuatro grandes problemáticas que afectan de manera directa sus derechos.

El primer antecedente de lo que hoy se conoce como la moratoria social, es el que nace de una de las movilizaciones sociales más representativas de la historia, la Revolución Industrial (Kustrin, 2007). Como es conocido, la Revolución industrial propició las primeras regulaciones laborales, gracias al movimiento obrero que buscaba mejores condiciones laborales, entre ellas, las relacionadas con el tratamiento de las mujeres y los menores en las fábricas.

Una de las principales consecuencias de estos logros fue el cambio de condiciones de realidad para mujeres y niños, que mejoró ostensiblemente sus condiciones de existencia. Pero, para el caso que nos ocupa, uno de los fenómenos más importantes fue la regulación que sacó a los niños de las fábricas en Europa, obligando a que permanecieran en los centros educativos por más tiempo, antes de que empezaran a trabajar. Así, en los años posteriores a la Revolución, se transformó profundamente la sociedad europea y se configuró la importancia de la capacitación, haciendo que los niños permanecieran cada vez más años en centros de formación académica, alejados del trabajo.

Este cambio es lo que conocemos hoy como moratoria social, en donde las poblaciones jóvenes están más tiempo en los centros educativos por periodos cada vez más extensos, permitiendo que exista una moratoria en la asunción de responsabilidades propias de los adultos, ya que los jóvenes permanecen temporadas mucho más extensas en los centros de formación académica. Además, los procesos de formación se han especializado y llegado a un punto de instrucción muy específica, y los niveles de profundidad son cada vez mayores, generando así, una de las primeras necesidades en la vida de la juventud: la educación.

Un segundo antecedente histórico es el que se relaciona con las guerras modernas, que han marcado con sangre las condiciones de juventud en todos los contextos sociales del siglo XX (Kustrin, 2007), dado que los periodos bélicos de 1914 y 1939 (Primera y Segunda Guerra Mundial) fueron alimentados por hombres jóvenes en los ejércitos, fueron ellos y sus familias los dolientes de estos sangrientos conflictos. 
Solo durante la Primera Guerra, se cree que fallecieron cerca de 10 millones de combatientes (Diario La Nación, 2014), miembros de los ejércitos en conflicto, dejando a su paso no solo sus muertes, sino huérfanos y miles de heridos y desaparecidos. Lo que obligó a que la sociedad se sumergiera en otra transformación que trastocó las costumbres, compeliendo a las mujeres a asumir roles de hombres, tras su ausencia como consecuencia de los conflictos. Esto hizo que las mujeres también cambiaran sus usos y costumbres, frente a las formas de trabajo, tiempo, asunción de responsabilidades y educación de los hijos. Se hace evidente la segunda necesidad y afectación de derechos relacionados con la vida.

Un tercer acontecimiento histórico que afectaría de manera notoria la condición de juventud fue la gran depresión de los años 30. Este periodo fue difícil para la población juvenil, en cuanto la falta de dinero para inversión pública vio como principal forma de ahorrar capitales, el retiro de recursos públicos para la educación, y salud pública para la población. Como resultado, los procesos de formación para jóvenes que mantenían en los centros educativos, herencia de esos logros sociales de la Revolución Industrial, fueron abruptamente interrumpidos (Kustrin, 2007).

Esto, aunado a la gran falta de empleo y las dificultades económicas, generó un momento particularmente difícil para los jóvenes de todo el mundo. Por una parte, la imposibilidad de mantenerse en el proceso de formación, y por otra, las dificultades para obtener empleo. Desde entonces y teniendo en cuenta las diferentes realidades económicas de los países, se configura la tercera necesidad representativa para la juventud, el trabajo.

Por último, dentro de este breve recuento de los puntos de inflexión en la historia de la juventud, encontramos uno que es necesario nombrar desde el primer momento, la participación. A lo largo de la historia del siglo XX, los jóvenes han hecho parte de las grandes movilizaciones, incluso desde mucho tiempo atrás. Por lo cual es posible afirmar que la población juvenil ha estado presente como partícipes activos y movilizadores sociales en los principales conflictos políticos y bélicos de Occidente. Un famoso ejemplo de esta afirmación fue el asesino del archiduque Fernando de Austria, joven estudiante nacionalista austrohúngaro perteneciente al movimiento "Joven Bosnia", en la Primera Guerra mundial.

De lo anterior, podemos observar que los grupos políticos conformados por jóvenes han estado presentes durante todo el siglo $\mathrm{XX}$, $\mathrm{y}$ que fueron miembros activos militantes de diversas posturas políticas. Muchas de ellas los han llevado al alistamiento militar, como a organizaciones clandestinas de diversos tipos y motivaciones políticas o contraculturales (Kustrin, 2007). 
Los años 50 y 60 son otro ejemplo de una explosión no solo cultural, sino de emancipación política y contrahegemónica, que criticó desde la guerra de Vietnam, hasta gobiernos dictatoriales, como en el caso de España, Argentina, Chile, Uruguay, México, y en años posteriores Perú, El Salvador, Guatemala, entre otros. Allí vemos que los principales actores políticos de estas movilizaciones, tanto en un bando como en el otro, eran movilizadores jóvenes, promotores y multiplicadores de ideas, como la revolución de los tenientes que tuvo lugar durante los años 20 (Sachi, 1971).

\section{El divorcio entre participación y movilización}

Para el caso de América Latina, sobre todo en el siglo XX, los acontecimientos que construyeron la historia de la juventud fueron marcados principalmente por la participación y los conflictos. A pesar de que los jóvenes y las juventudes han estado presentes en las movilizaciones sociales, la idea de juventud como la conocemos hoy, es decir, población diferenciada, con derechos y problemáticas propias, aparece en nuestro continente hasta los años 80 (Cepal, 1985).

Para el caso colombiano, la población juvenil surge solo hasta 1991 con la Constitución Política, en su Artículo 45. Empero, y reconociendo que esto significó un gran avance en el tema, mas aún, cuando siete años después aparece la primera regulación en temas de juventud propiamente dicha, la Ley 375 de 1997, que inició el camino de la adopción del discurso de la participación juvenil, aunque este nacería con serias contradicciones. Si nos regresamos un poco en el siglo XX, las movilizaciones sociales relacionadas con los conflictos políticos y económicos eran movilizaciones que contenían la fuerza popular y representaron la vida en relación con las necesidades populares.

Basta con recordar sobre las movilizaciones estudiantiles, las cuales se hicieron una con la unión de las formas de lucha, haciendo de la juventud parte imprescindible de su esencia. Es posible afirmar que la idea de juventud se asociaba con la movilización estudiantil durante el siglo XX en toda América Latina, al contrario de lo que hoy sucede, y a partir de 1991, la juventud se asocia a la juventud misma, escindiendo profundamente el discurso de la participación del discurso de la movilización.

Esto representa una ruptura esencial y manifiesta, que diferencia el siglo XX del siglo XXI. Pero esta ruptura no es muestra de un avance en la construcción del discurso de los derechos y de la participación, por el contrario, ha representado una de las tantas formas que se han convertido en los adalides de la democracia, sin mayores resultados en la calidad de vida de las poblaciones, en este caso, la juventud. Si bien es cierto, es deber reconocer que la participación, vista desde el discurso esencial de la democracia y la defensa, no solo de las libertades, sino de la 
negación de todo aquello que pretende negarla, busca el ideal de la real inclusión de la población. Pero visto desde la perspectiva de la ruptura entre participación y movilización, este discurso oficial no ha aportado mayores logros en el tema.

En este orden de ideas, la idea oficial lo único que ha logrado es despersonalizar la movilización como mecanismo de búsqueda y defensa de los derechos, en una simple retórica en la que la juventud ya no se entiende como parte de sus propios problemas sociales, que responden a un desarrollo histórico político, económico y social. Si observamos los discursos de participación después de 1991, en especial el promovido por el Plan de Desarrollo de 2003 que crea Colombia Joven, estos promueven una participación juvenil, que hace una clara diferenciación entre juventud y estudiantes, consiguiendo así romper la herencia del movimiento estudiantil y crear una nueva intención de la participación juvenil más "moderna", que buscaría la real inclusión de la población en las agendas políticas y económicas, hecho que nunca sucedió.

Por el contrario de lo que se pretendía en el discurso, el rompimiento creó dos frentes de participación, uno histórico, asociado a la movilización y a la unión y creación de tejido social, a uno diferenciado que, en sus propias palabras, se debía interpretar como "autónomo", pero que en esencia carecía de un reconocimiento de los logros sociales de todo el siglo XX. Así las cosas, se propiciaron las circunstancias con las que se trataría a la población juvenil, y en las cuales se afincarían estereotipos con los que se ha juzgado en nuestros días.

Ideas como, el joven en búsqueda de identidad, el joven peligroso, el joven futuro del país, entre muchas otras, y que responden a corrientes de pensamiento por las que ha trasegado teóricamente la población, han dirigido los lineamientos de las diferentes políticas de juventud. Adicionalmente, la falta de seriedad con que se ha visto el tema y la carencia de recursos son muestra de la insuficiencia de inclusión e incidencia del discurso de participación juvenil que conocemos desde los años 90. Como se afirmó anteriormente, el rompimiento que originan los dos frentes de participación, los cuales propician esa división que despersonalizó la movilización, restó fuerza a la inclusión del tema juvenil en las agendas políticas.

Infortunadamente, esta división también trajo consigo el olvido o, en otras palabras, se acompaña de una memoria histórica conveniente y cargada en ciertos aspectos de la doctrina de la seguridad nacional. Esto permitió potenciar las visiones punitivas populistas, facilitando entregarle el rótulo de enemigos del sistema a quienes pretenden proponer un punto de vista distinto al del discurso oficial. Dentro de estas propuestas punitivas populistas encontramos disminuir cada vez más la edad para los adolescentes infractores de la ley penal, los toques de queda para menores de 
edad en diferentes ciudades del país, las sanciones sociales y etiquetamiento a la población joven perteneciente a comunidades o grupos contraculturales.

\section{El joven heredero y el discurso oficial}

Regresando al tema de la participación y observando desde una perspectiva histórica, las agendas de las movilizaciones sociales del siglo XX son, en esencia, las mismas consignas de reivindicación de los jóvenes que hacen parte del sistema de participación juvenil, dentro del discurso creado a partir de 1991. A su vez, estas consignas de reivindicación se han entregado a las formas de participación oficiales, creadas para que los jóvenes busquen oportunidades en un sistema que los ha negado desde antes de su concepción. Se presenta, entonces, la idea del joven heredero (Margulis \& Urresti, 1998), del joven que encarna la institucionalidad y las prácticas tradicionales, aquel que es en este discurso el adalid de la democracia que se inserta en las organizaciones políticas, reproduciendo no solo el discurso, sino sus costumbres. Un joven que, en esencia, es un joven envejecido (Ariza, 2011).

Lo anterior invita a evidenciar una dificultad, que nace de la concepción misma de juventud, dificultad que consiste en el desconocimiento de la esencia de los jóvenes como población y movilizador social, en el discurso oficial de participación. Esto potenciado por la carencia de normas orientadas a la apertura del espectro participativo, que en el universo de la juventud es absolutamente necesario y diverso, para lograr materializar la tan anhelada inclusión real y efectiva.

Otro punto que genera limitaciones en el tema que nos reúne es la dificultad que ha representado para las instituciones abordar el tema de la diversidad. La población juvenil es en sí misma una manifestación de diversidad, dentro de las múltiples formas de ver el mundo. Visto esto no solo desde la amplitud que supone la población en términos de ciclo vital, sino de cosmovisiones ancestrales, sea por herencia directa o por autorreconocimiento, en el caso de las poblaciones indígenas, comunidades afrodescendientes, raizales, campesinos. Además de comunidades religiosas, que tienen en sus grupos de jóvenes una fuerza importante, como los rastafari, o las comunidades cristianas o evangélicas, entre muchos ejemplos de coexistencia de la multiplicidad de conciencias.

También, las diferencias que se presentan en cuanto a momentos de vida, como los jóvenes que están vinculados al sistema educativo, o los que están en el mercado laboral, o desempleados. También, las diferencias que supone la asunción de las responsabilidades en el cambio de un tipo de familia a otro, o las diferencias que se presentan en relación con el género o la orientación sexual. 
Aunque en las dificultades que se han hecho visibles dentro del discurso de participación, no es cierto que el problema sea la participación en sí misma, ya que eso sería desconocer un sinnúmero de procesos exitosos, o líderes que han intentado hacer la diferencia entre la idea oficial y la movilización social. Pero no debe negarse que el discurso oficial se ha valido de una imagen de joven que hereda las tensiones tristes de la tradicionalidad, y estas se han incrustado en el sistema que se ha impuesto y defendido con el tiempo. Los jóvenes interesados en hacer parte del sistema, o con inquietudes comunitarias, desean ser como los políticos tradicionales, o intentan ser diferentes, pero sus posturas políticas son las mismas del discurso.

Esto perpetúa las carencias en los procesos de participación, desconociendo no solo esas características diversas de la juventud y los grupos o manifestaciones en el interior de la población, sino la idea de juventud en sí misma, ya que el discurso de la participación solo se ha detenido a conglomerar a los jóvenes y no a comprender sus procesos y realidades, requisito sin el cual no es posible construir una verdadera participación.

La idea del joven heredero distancia a los jóvenes que no se reconocen en esa propuesta institucional, pues consideran cimentar sus actuaciones de construcción del tejido social, lejos de la institucionalidad. Las consecuencias principales de la juventud lejana de la institucionalidad, además de demostrar una falta de confianza en las instituciones, es el fracaso de los procesos de participación juvenil, que hace necesaria e inevitable una nueva interpretación del concepto, que se explique y se resignifique desde la realidad juvenil.

Estos procesos fracasan porque no son pensados para la juventud, ya que los mismos nacen de los mecanismos tradicionales que, en gran medida, no recogen su realidad social, y con mayor razón en el contexto de realidad colombiana. Si bien es necesario rescatar y reivindicar los valores de la democracia, como un pilar del Estado social de derecho, que hace de la participación una herramienta, también es necesario que estos valores dejen de interpretarse con los vicios de la participación del siglo XX en Colombia. Vicios que representan el odio, la violencia, la poca representatividad que tienen las verdaderas necesidades sociales en las altas decisiones de política económica y social.

Además, la resistencia al cambio y la resistencia a la diferencia, que es una herencia propia del maniqueísmo religioso y de la idiosincrasia ortodoxa, se erigen como una barrera cultural y espiritual, que nos detiene en el proceso de la construcción de la inclusión real, que usa la participación como herramienta de afianzamiento de 
la democracia y, por ende, la materialización de ese tan anhelado Estado social de derecho.

\section{Conclusión}

Es necesario replantear el significado de la participación y el ejercicio de los mecanismos dirigidos a la población juvenil, ya que este es solamente el primer paso para el verdadero reconocimiento de la población juvenil y, de esta manera, abonar el camino para vindicar los derechos humanos de los jóvenes en Colombia.

\section{Referencias}

Angarita, C. (2010) La rebelión del sujeto y los derechos humanos. En C. Angarita (Ed.). Teoría crítica de los derechos humanos (pp. 97 - 125). Tunja, Colombia: Salamandra Grupo Creativo.

Ariza, N. (2011). El joven político y la vida política del joven en Boyacá. Debate prospectivo sobre la educación politica de la población joven y efectos en el desarrollo regional. Tesis de Maestría en Estudios Políticos. Pontificia Universidad Javeriana. Bogotá Colombia. Recuperado de http://repository. javeriana.edu.co/bitstream/10554/1427/1/ArizaBarbosaNilce2011.pdf

Rodríguez, E. (2004). Políticas públicas de juventud en América Latina. Empoderamiento de los jóvenes, enfoques integrados, gestión moderna y perspectiva generacional. En Construcción de políticas públicas de juventud. Análisis y perspectivas (pp. 77-115). Manizales, Colombia: Universidad de Manizales, Programa Presidencial Colombia Joven, Centro de Estudios Avanzados en Niñez y Juventud Cinde, Universidad de Manizales, GTZ, Unicef.

La Nación. (2014, 11 de feb.). La Primera Guerra Mundial en cifras. Recuperado de http://www.lanacion.com.co/index.php/component/k2/item/230069-laprimera-guerra-mundial-en-cifras Observado 1 de Abril de 2016.

Estudios e Informes de la Cepal (1985). La juventud en América Latina y el Caribe. Plan de acción regional en relación con el Año Internacional de la Juventud. Santiago de Chile: Ediciones y Publicaciones ONU.

Kustrin, S. (2007, 15 de jun.). Juventud teoría e historia: la formación de un sujeto social y un objeto de análisis. Historia Actual OnLine. Recuperado de https:// dialnet.unirioja.es/descarga/articulo/2479343.pdf 
Sachi, H. (1971). Luis Carlos Pestes, y la larga marcha de 1922. Facultad de Ciencias Sociales UBA. Recuperado de: http://www.sociales.uba.ar/wpcontent/uploads/12-Sacchi.pdf

Margulis, M. \& Urresti, M. (1998). La construcción social de la condición de juventud. En Viviendo a toda: jóvenes, territorios culturales y nuevas sensibilidades (pp. 3-20). Bogotá: Universidad Central -DIUC-, Siglo del Hombre Editores.

Montenegro, A. (1987). Historia del Antiguo Continente. Bogotá: Norma.

Quintero, F. (2014). Jóvenes, participación, representaciones y ciudadanía. En Diplomado Paz a Tiempo, Universidad Santo Tomás. Recuperado de: http:// soda.ustadistancia.edu.co/enlinea/pazatiempo/eje1/mod1/unidad3/Jovenes Participacion.pdf 